\title{
Therapeutic Potential of Probiotics -Lactobacillus Plantarum UBLP40 and Bacillus Clausii UBBC07 on Thioacetamide-Induced Acute Hepatic Encephalopathy in Rats
}

Chirag Amrutlal Patel ( $\nabla$ chiragapatel@lmcp.ac.in )

LM College of Pharmacy https://orcid.org/0000-0003-2969-2955

Lalita Shahgond

SSR College of Pharmacy Department of Pharmacology

Khushboo Thakur

SSR College of Pharmacy Department of Pharmacology

Dipta Sarkar

SSR College of Pharmacy Department of Pharmacology

Sanjeev R Acharya

SSR College of Pharmacy Department of Pharmacology

Priyanshi Patel

SSR College of Pharmacy Department of Pharmacology

\section{Research Article}

Keywords: Hepatic Encephalopathy, Probiotics, Lactobacillus plantarum, Bacillus clausii, Thioacetamide

Posted Date: March 31st, 2021

DOl: https://doi.org/10.21203/rs.3.rs-359488/v1

License: (c) (1) This work is licensed under a Creative Commons Attribution 4.0 International License.

Read Full License 


\section{Abstract}

Purpose: Hepatic encephalopathy (HE) or hepatic coma is an onerous, not utterly understood complication accompanying both acute and chronic liver dysfunction and/ or presence of portosystemic shunting. In HE, hyperammonemia and inflammatory responses are believed to act in synergism. Probiotics, Lactobacillus plantarum UBLP40 and Bacillus clausii UBBC07 reduce small intestinal bacterial overgrowth and hyperammonemia, thereby preventing the development of $\mathrm{HE}$.

Methods: The effect of probiotics-Lactobacillus plantarum UBLP40 ( $10^{7} \mathrm{CFU} /$ day, 14 days) and Bacillus clausiUUBBC07 (107 CFU/day, 14 days) combination and standard drug-lactulose $(2.5 \mathrm{ml} / \mathrm{kg}$ in 3 divided doses, 14 days) was studied in thioacetamide $(250 \mathrm{mg} / \mathrm{kg}$ for 3 days) induced acute HE in rats by measuring behavioural parameters, biochemical parameters (serum AST, ALT, ALP and ammonia level), neurochemical parameters and histopathology study in brain and liver.

Results: In contrast to only thioacetamide treated rats, probiotics treatment substantially $(p<0.001)$ reduced liver function parameters i.e. serum AST, ALT, ALP, and ammonia, improved behaviour parameters, i.e. decreased motor disruption, improved memory impairment. Probiotics treated rats have also shown a substantial improvement in parameters of oxidative stress, i.e. decreased lipid peroxidation and increased glutathione level in brain tissue and ameliorate the histopathological changes induced by thioacetamide in brain and liver.

Conclusions: It can be concluded based on the findings that the combination therapy of Lactobacillus plantarum UBLP40 and Bacillus clausiUBBC07 proves to be effective in acute hepatic encephalopathy in the preclinical stage and further studies are required to assess this therapy potential in the clinical stage.

\section{Introduction}

Hepatic encephalopathy (HE) or hepatic coma is an onerous, not utterly understood complication accompanying acute, chronic liver dysfunction and/ or portosystemic shunting (Khungar and Poordad 2012; Prakash and Mullen 2010). Its symptomatic signs may range from minor behavioural changes to coma, in severe conditions it may lead to death (Prakash and Mullen 2010). HE has proven to be the most significant concern to patients with liver disorders (Cooper 2001; Ferenci 2017; Savy et al. 2018).

Several theories have been proposed explaining the causes behind the development of HE and its progression. Though, the actual mechanism underlying behind HE is still under debate (Azhari and Swain 2018; Elwir and Rahimi 2017; Hadjihambi et al. 2018; You et al. 2017). The hypothesis generated says about the role of 'ammonia' (NH3) and other neurotoxins which damaged liver does not excrete and is stowed in the humans (Azhari and Swain 2018; Hadjihambi et al. 2018; Parekh and Balart 2018). Gut microflora species are accountable for the generation of $\mathrm{NH} 3$ from various nitrogen sources as well by the enterocytes (Diether and Willing 2019). Physiologically, ammonia enters the brain and is utilized by astrocytes to produce glutamine from glutamate (Suarez et al. 2002). This mechanism is dominant in the case of impaired liver function. As the glutamine level gets elevated in the astrocytes cells to 
counterbalance excess solute due to osmotic gradient, fluid enters in the cells (Cooper 2001). This leads to fluid accumulation which causes brain herniation and finally death. Hyperammonemia also stimulates oxidative stress and inflammation (Cooper 2001; Savy et al. 2018).

Probiotics have always been proved to improve human health in various diseased conditions and some research of it is found in the treatment of hepatic encephalopathy (Liu et al. 2018). Lactobacillus plantarum UBLP40 is extracted from milk products and fermented foods, useful for maintaining the acidity in the gut region. Bacterial induced acidic medium traps the ammonium ion and reduces hyperammonemia (da Silva Sabo et al. 2014). Bacillus clausii UBBC07, a spore-forming bacterium that exhibits resistance to commonly used antibiotics, intestinal wall adhesion, secretion of anti-microbial against Gram-positive bacteria and immunomodulatory response, is another probiotic (Duc et al. 2004). This probiotic is also indicated in conditions like diarrhea and Small intestinal bacterial overgrowth (SIBO) (Chen and Quigley 2014). We extend our hypothesis that probiotics may reduce SIBO and hyperammonemia, thereby preventing the development of HE. In this study, we evaluated the effect of potential probiotic (Lactobacillus plantarum UBLP40 and Bacillus clausii UBBC07) against thioacetamide (TAA) induce hepatic encephalopathy. TAA mainly damages the hepatocytes which carry out the urea cycle and halts the metabolism of ammonia (Chen et al 2008; Farjam et al, 2012).

\section{Materials And Methods}

\subsection{Probiotic}

The strain of Lactobacillus plantarum UBLP40 and Bacillus clausii UBBC07 was obtained from Unique Biotech Ltd, India. Certificate of Analysis of both strains included in supplementary file 1. It was inoculated in sterilized MRS broth solution and incubated at $37^{\circ} \mathrm{C}$ in the bacteriological incubator. Bacterial species were pelleted by centrifugation at 2000-3000 rpm, organisms were re-suspended in 500 $\mathrm{mL}$ phosphate buffered saline (PBS, $\mathrm{pH7.4}$ ) and administered in rats via oral gavage every day.

\subsection{Acid resistance, Bile resistance and Bile salt hydrolase (BSH) activity:}

Both the strain of probiotic Lactobacillus plantarum UBLP40 and Bacillus clausiiUBBC07 subjected for evaluation of its acid resistance, bile resistance and BSH activity as described by Patel et al. (2020).

\subsection{Animals and Housing}

The SD rats weighing 170-200 gm were obtained from the Bombay Veterinary College, department of pharmacology and toxicology, Maharashtra, India. The study protocol was approved by the IAEC (No: SSR/IAEC/2019/05). The study was conducted as per the CPCSEA guide for the laboratory animals. Twenty-four rats were housed and maintained under the standard condition in the good laboratory practice (GLP) certified animal house facility at SSR College of Pharmacy, India.

\subsection{Experimental Design}


The SD rats were divided into four groups $(n=6)$ and were acclimatized for a period of 1 week. After 7 days of acclimatization span,

- Group I was assigned as a negative control

- Group II served as induced control which was treated with thioacetamide $(250 \mathrm{mg} / \mathrm{kg} /$ day i.p at an interval of $24 \mathrm{~h}$ for 3 days),

- Group III was treated with thioacetamide (250 mg/kg/day i.p, 3 days) + Lactobacillus plantarum UBLP40 (107 CFU/day, p.o for 14 days) + Bacillus clausii UBBC07 (10 $\mathrm{CFU} /$ day, p.o for 14 days)

- Group VI was given thioacetamide $(250 \mathrm{mg} / \mathrm{kg} /$ day i.p at an interval of $24 \mathrm{~h}$ for 3 days) + Standard treatment i.e lactulose ( $2.5 \mathrm{ml}$ per $\mathrm{kg}$ in 3 divided doses, p.o for 14 days).

On the $14^{\text {th }}$ day, rats were tested for behavioural functions (after two hours of treatment). On the $15^{\text {th }}$ day, rats were sacrificed, initially, blood was collected for the determination of biochemical parameters and then liver and brain samples were collected for determination of neurochemical parameters and histopathological studies (Fig 1).

\subsection{Behavioural tests}

\subsubsection{Rotarod test}

An accelerating rotarod instrument was employed to record motor coordination, as per Vijitruth et al. (2018) method. Rats were trained and the basal falling latency was recorded. After treatment, the final falling latency was recorded.

\subsubsection{T-maze test}

The cognitive ability of the rats was analysed using T- shaped maze. This test was performed according to the procedure stated by Hussein et al. (2018). Firstly, the animals are subjected to habituation period at intervals; this is a crucial stage where the animals will inculcate learning only through habituation. Secondly, they are rewarded properly based on how they act and what path they choose during the habituation process and then trained accordingly. Spontaneous alternation is the main working principle. After placing the rat at the entrance of $t$-maze for $10 \mathrm{~s}$, the barrier was raised and the animal was permitted to travel the maze for 30 seconds. It was granted a free choice. Thus, when it entered one of the arms, it was blocked for 30 seconds, to explore this place. Afterwards, it was kept again at the starting point. The session was restarted if the rat didn't choose either of the arms after the 30s. A correct response is "an alternating choice between the two arms". The time of response (seconds) is also recorded. Is there any latency in entering the goal arms or not and if yes then how much?

$\%$ of Alternation $=\frac{\text { number of correct response }}{\text { total number of sessions }}$

\subsubsection{Object recognition test}


The design of test apparatus was as per Ennaceur and Delacour (1988). Two trials T1 and T2 were done 2-min each. In T1 (two identical objects) trial and T2 (one identical object was replaced with the unknown object), trial objects were placed in two opposite corners of the in which the rats were to be placed. After placing the rat into the ORT apparatus, it was allowed to explore the objects. Trial T2 was performed after 1-hour trial T1. Exploration was defined as follows: "directing the nose toward the object at a distance of not more than $2 \mathrm{~cm}$ and/or touching the object with the nose".

\subsection{Biochemical parameters}

\subsubsection{Serum Alanine aminotransferase (ALT), Aspartate aminotransferase (AST) and Alkaline Phosphatase (ALP)}

The blood sample was centrifuged at $1500 \times \mathrm{g}$ at $4 \mathrm{C}^{\circ}$ for 15 minutes. The serum was deliberately isolated and used for further testing (El-Marasy et al. 2019). Assay kits based on colorimetric method were utilized to detect ALT and AST. pNPP- AMP kinetic assay method kit was utilized to quantify the amount of ALP in serum.

\subsubsection{Serum Ammonia}

This technique is based on the formation of indophenol which is a blue color compound and should be this color is persistent. The testing procedure was performed as per Muramatsu (1967). This method comprises two steps: firstly, treatment of blood and second development of color. The optical density of the established color was recorded at $610 \mathrm{~nm}$.

\subsection{Neurochemical parameters}

\subsubsection{Brain tissue homogenization}

In $20 \%$ phosphate buffer saline (PBS), the brain tissue was homogenized, centrifuged for 15 minutes at $1500 \mathrm{~g}$ at $4 \mathrm{C}^{\circ}$ and the supernatant was obtained for direct measurement of oxidative stress parameters (El-Marasy et al. 2019).

\subsubsection{Lipid peroxidation}

TBARS method of Ruiz-Larrea et al. (1994) was used to determine lipid peroxides in the brain of rats. A pink colored component was formed after mixing the reactants and heating it for 30 minutes. The absorbance of this compound was measured at $532 \mathrm{~nm}$.

\subsubsection{Reduced glutathione}

The content of reduced glutathione in brain tissue was measured using Ellman's reagent (DTNB) (Danduga et al. 2018) Centrifugation was done for $20 \mathrm{~min}$ to separate supernatant of the mixture [Equal volumes of tissue homogenate and TCA $(10 \%)]$. In $1 \mathrm{ml}$ of supernatant $3 \mathrm{ml}$ of phosphate buffer $(0.2 \mathrm{M}, \mathrm{pH}$ 
8) and $0.5 \mathrm{ml}$ of DTNB reagent $(0.6 \mathrm{mM}$ in $0.2 \mathrm{M}$ Phosphate buffer) were added and mixed after which absorbance was recorded at $412 \mathrm{~nm}$.

\subsection{Histopathological study of brain and liver}

After withdrawing blood samples, the rats were euthanized. Liver and brain from different group rats were isolated and fixed in $10 \%$ neutral buffered formalin. These samples were sent for histopathological slide and paraffin wax preparation to Sakshi Histopathology Works, India. The prepared slides were observed under the microscope, images were captured. The injury in liver cells, as well as brain cells injury and inflammation, were determined.

\subsection{Statistical analysis}

This analysis was done using Graph Pad Prism 8 (software, India). The results were indicated as mean $\pm S E M$. All the data were analyzed by a one-way ANOVA test with a significance level $(P<0.05)$.

\section{Results}

\subsection{Acid Tolerance, Bile Salt Tolerance, and BSH Activity}

This result of test indicates that B. clausii UBBC07 and Lact. Plantarum UBLP40 were able to withstand and survive in highly acidic conditions and intestinal fluids.

\subsection{Behavioural tests}

\subsubsection{Rotarod test}

The effect of TAA and the probiotics on motor coordination is illustrated in Fig 2. This data reveals the fall time of TAA administered rats (Group II) was significantly reduced compared to the negative control group. The probiotic and lactulose treated rats showed improved motor control and muscle grip strength compared to Group II rats (3-NP) on the fourteenth day of the treatment regimen.

\subsubsection{T-maze test}

The TAA treated animals showed longer transfer latency than vehicle-treated normal rats $(P<0.001)$ indicating impairment of memory (Fig 3). Probiotic treatment substantially reduced memory impairment in the treated group $(P<0.01)$ by reducing transfer latency relative to Group II animals on the 14th day of the treatment regimen.

\subsubsection{Object recognition test}

The rats induced with TAA were not able to distinguish between the new and familiar object as compared to the negative control group (Fig 4). 14-day probiotic treated rats significantly distinguish new and 
familiar object and explored more time with new object compared to induced control, demonstrating that reduced memory impairment caused by TAA.

\subsection{Biochemical assessment}

\subsubsection{AST and ALT and ALP}

The results of the liver function test (AST, ALT and ALP test) showed that TAA caused a substantial increase in serum AST, ALT and ALP levels relative to the negative control group (Table 1) suggesting marked liver damage. Treatment with probiotics significantly decreases the serum AST, ALT and ALP level compared to induced control, suggesting that the probiotic treatment was able to protect against TAArelated injury.

\subsubsection{Serum ammonia}

The TAA administration resulted in a large rise in ammonia levels in the blood of rat's relative to the negative control group. Treatments with probiotic and lactulose showed significantly low ammonia level as compared to the induced control group (Table 1).

\subsection{Neurochemical Assessment}

\subsubsection{Lipid peroxidation}

The administration of TAA culminated in a substantial rise of lipid peroxidation in the brain, suggesting damage incurred by oxidative stress (Table 1). Rats that obtained probiotic and lactulose therapy reported a substantial decrease in TAA-induced lipid peroxidation, suggesting a pronounced reduction in oxidative stress in brain tissues.

\subsubsection{Reduced Glutathione}

Treatment with TAA increased the oxidative stress, as evidenced by the significant decrease in GSH levels compared with the control group (Table 1). The probiotic and lactulose-treated group reported a large improvement in GSH levels relative to the TAA group.

\subsection{Histopathology study:}

The microscopy was performed under 50x, 200x, 400x to examine the abnormalities in different organs and cells. The livers of rats in the control group (Fig 5IC) showed normal histological structures. The cells like hepatocytes and kupffer cells were intact and no inflammation was observed. The livers from rats in the TAA group (Fig 5 IIC) showed damaged liver cells (hepatocytes and kupffer cells) and slight inflammation. In disparity, the rats treated with probiotic (Fig 5 IIIC) exhibited little damage but the cells were intact as compared with group IIA. However, treatment with lactulose showed no sight of damage and inflammation (Fig 5 IIIC). 
In brain's histology section, the hippocampus region was observed. The neurons and glial cells were observed especially astrocytes. In induced control group Alzheimer type II astrocytes were not visible (Fig 6 IIC). Group III (Fig 6 IIIC) and group IV rats (Fig 6 IVC) showed normal histological structures, similar architecture of hippocampus as compared to group I.

\section{Discussion}

Our current study represents the protective prospect of probiotics in acute hepatic encephalopathy, which is regarded as a complication of liver damage. Astrocytes swelling or Alzheimer type II astrocytes are considered as the hallmark of HE (Agarwal and Mais 2019). Liver damage in combination with increased serum ammonia level and release of inflammatory cytokines into systemic circulation might be the possible factors for such hallmark (Swaminathan et al. 2018).

TAA induced fulminant hepatic encephalopathy is widely used preclinical models in laboratories as these models represent similar characteristics of HE in rats. Hepatotoxic nature of TAA aids to degenerate the hepatocytes, where the urea cycle conducts ammonia metabolism leading to the insufficient liver (Auron and Brophy 2018). Hence, increasing the serum ammonia level, inflammation-causing astrocyte swelling and various other clinical manifestation such as cognitive impairment, motor coordination and sleep disturbances (Elwir and Rahimi 2017; Ferenci 2017). In our present study, crucial hepatic necrosis, swelled astrocytes and vacuolization, markers of HE were observed in histopathological study of the liver in TAAinduced $\mathrm{HE}$ rats that indicated morphological abnormalities as a result of hyperammonemia, oxidative stress and neuro-inflammation (Jayakumar et al. 2015). The standardization of the TAA model (Data are not shown) performed to fix the dose of TAA with less mortality and higher liver damage. So, the dose of $250 \mathrm{mg} / \mathrm{kg}$ was fixed from the doses of $225 \mathrm{mg} / \mathrm{kg}, 250 \mathrm{mg} / \mathrm{kg}$ and $275 \mathrm{mg} / \mathrm{kg}$.

In the present study, ingestion of TAA at a dose of $250 \mathrm{mg} / \mathrm{kg}$ for three days in rats resulted in a significant reduction of the final falling latency duration in the Rotarod test. The possible reason for this observation is the activation of cerebral astrocytes and microglia in brain due to increased blood ammonia level causing impairment of motor coordination via neuro-inflammation chaperoned by heightened levels of gamma amino butyric acid in the cerebellum (Aldridge et al 2015; Jaeger et al. 2019). Final falling latency was elevated on probiotic administration as it improved the motor coordination. Another behavioral parameter cognition and memory were evaluated by ORT, here administration of TAA impaired the cognition as the rats were not able to distinguish between the novel object and familiar object but memory improvement was observed on administering the combination therapy as the rats were able to distinguish (Ennaceur and Delacour 1988). The T-maze test was also executed to access the memory of rats that were having TAA induced HE. Response time was elevated while the percentage of alternation was decreased in rats as the memory and motor coordination was impaired (Hussein et al. 2018). Improvement was observed in rats after administration of probiotic treatment. 
Escalated liver enzymes concentration in the serum indicated leaching of AST, ALT and ALP directly into the bloodstream from the degenerated liver cells showing remarkable liver damage in only TAA treated rats (Giannini et al. 2005). The rise in blood ammonia level was also observed in TAA administered group while ammonia level was lesser in probiotics treated group. The ammonia liberated from injured liver cells were prevented from being absorbed from the small intestine by probiotic. The site of action of probiotics is small intestine where they are responsible for acidification of gut. Acidic medium converts ammonia into ammonium ion which gets trapped into the gut region and never crosses the gut membrane as well as blood brain barrier (Ballan et al. 2020).

Normally GSH is a brain's free radical scavenger and its level is decreased in TAA treated rats indicating generation of more free radicals ( $\mathrm{Li}$ et al. 2015). Lipid peroxidation level was increased as the degeneration of lipids in the brain was raised by free radical. These free radicals were stimulated by hyperammonemia and are accompanied by astrocyte swelling. This way, in TAA-induced HE showed a higher MDA level which results in formation of free radicals leading to oxidative damage and reduced brain GSH content. Rats that treated probiotic therapy reported a substantial decrease in TAA-induced lipid peroxidation and increase GSH content, suggesting a pronounced reduction in oxidative stress in brain tissues (Danduga et al. 2018; Ruiz-Larrea et al. 1994).

\section{Conclusion}

Based on the results and discussion of this study it can be concluded that the combination therapy of Lactobacillus plantarum UBLP40 and Bacillus clausii UBBC07 proves to be effective in acute hepatic encephalopathy in preclinical stage. Therefore, further studies are required to assess this therapy potential in clinical stage.

\section{Declarations}

Funding: No funds, grants, or other support was received.

Conflicts of interest/Competing interests: The authors have no conflicts of interest to declare that are relevant to the content of this article.

Availability of data and material (data transparency): All data generated or analyzed during this study are included in this published article [and its supplementary information files].

Author Contribution Statement: The authors declare that all data were generated in-house and that no paper mill was used

Ethics approval: IAEC (Institutional Animal Ethics Committee) approved the experimental protocol SSR/IAEC/2017/05.

Consent to participate: Not applicable 
Consent for publication: Authors give consent for information to be published in the journal- NaunynSchmiedeberg's Archives of Pharmacology

\section{Acknowledgements: NA}

\section{Author Contribution Statement}

Conceptualization: [Chirag Patel, Lalita Shahgond, Sanjeev Acharya, Priyanshi Patel]; Methodology: [Lalita Shahgond, Khushboo Thakur, Dipta Sarkar]; Formal analysis and investigation: [Chirag Patel, Lalita Shahgond, Khushboo Thakur, Dipta Sarkar]; Writing - original draft preparation: [Chirag Patel, Lalita Shahgond, Priyanshi Patel]; Writing - review and editing: [Chirag Patel, Lalita Shahgond, Sanjeev Acharya]; Supervision: [Sanjeev Acharya]

\section{References}

1. Agarwal AN, Mais DD (2019) Sensitivity and Specificity of Alzheimer Type II Astrocytes in Hepatic Encephalopathy. Arch Pathol Lab Med 143(10):1256-1258. doi:10.5858/arpa.2018-0455-OA

2. Aldridge DR, Tranah EJ, Shawcross DL (2015) Pathogenesis of hepatic encephalopathy: role of ammonia and systemic inflammation. J Clin Exp Hepatol 5(Suppl 1):S7-S20. doi:10.1016/j.jceh.2014.06.004

3. Auron A, Brophy PD (2012) Hyperammonemia in review: pathophysiology, diagnosis, and treatment. Pediatr Nephrol 27(2):207-222. doi:10.1007/s00467-011-1838-5

4. Azhari H, Swain MG (2018) Role of Peripheral Inflammation in Hepatic Encephalopathy. J Clin Exp Hepatol 8(3):281-285. doi:10.1016/j.jceh.2018.06.008

5. Ballan R, Battistini C, Xavier-Santos D, Saad SMI (2020) Interactions of probiotics and prebiotics with the gut microbiota. Prog Mol Biol Transl Sci 171:265-300. doi:10.1016/bs.pmbts.2020.03.008

6. Chen TM, Subeq YM, Lee RP, Chiou TW, Hsu BG (2008) Single dose intravenous thioacetamide administration as a model of acute liver damage in rats. Int J Exp Pathol 89(4):223-231. doi:10.1111/j.1365-2613.2008.00576.x

7. Chen WC, Quigley EM (2014) Probiotics, prebiotics \& synbiotics in small intestinal bacterial overgrowth: opening up a new therapeutic horizon! Indian J Med Res 140(5):582-584

8. Cooper AJ (2001) Role of glutamine in cerebral nitrogen metabolism and ammonia neurotoxicity. Ment Retard Dev Disabil Res Rev 7(4):280-286. doi:10.1002/mrdd.1039

9. da Silva Sabo S, Vitolo M, González JMD, Oliveira RPS (2014) Overview of Lactobacillus plantarum as a promising bacteriocin producer among lactic acid bacteria. Food Res Int 64:527-536. doi:10.1016/j.foodres.2014.07.041

10. Danduga RCSR, Dondapati SR, Kola PK, Grace L, Tadigiri RVB, Kanakaraju VK (2018) Neuroprotective activity of tetramethylpyrazine against 3-nitropropionic acid induced Huntington's disease-like symptoms in rats. Biomed Pharmacother 105:1254-1268. doi:10.1016/j.biopha.2018.06.079 
11. Diether NE, Willing BP. Microbial Fermentation of Dietary Protein: An Important Factor in Dietâđ» Microbeâđ»Host Interaction. Microorganisms. 2019 Jan 13;7(1):19. doi:

10.3390/microorganisms7010019

12. Duc le H, Hong HA, Barbosa TM, Henriques AO, Cutting SM (2004) Characterization of Bacillus probiotics available for human use. Appl Environ Microbiol 70(4):2161-2171. doi:10.1128/aem.70.4.2161-2171.2004

13. El-Marasy SA, El Awdan SA, Abd-Elsalam RM (2019) Protective role of chrysin on thioacetamideinduced hepatic encephalopathy in rats. Chem Biol Interact 299:111-119. doi:10.1016/j.cbi.2018.11.021

14. Elwir S, Rahimi RS (2017) Hepatic Encephalopathy: An Update on the Pathophysiology and Therapeutic Options. J Clin TransI Hepatol 5(2):142-151. doi:10.14218/JCTH.2016.00069

15. Ennaceur A, Delacour J (1988) A new one-trial test for neurobiological studies of memory in rats. 1: Behavioral data. Behav Brain Res 31(1):47-59. doi:10.1016/0166-4328(88)90157-x

16. Farjam M, Dehdab P, Abbassnia F, Mehrabani D, Tanideh N, Pakbaz S, Imanieh MH (2012) Thioacetamide-induced acute hepatic encephalopathy in rat: behavioral, biochemical and histological changes. Iran Red Crescent Med J 14(3):164-170

17. Ferenci P (2017) Hepatic encephalopathy. Gastroenterol Rep (Oxf) 5(2):138-147. doi:10.1093/gastro/gox013

18. Giannini EG, Testa R, Savarino V (2005) Liver enzyme alteration: a guide for clinicians. CMAJ;172(3):367 - 79. doi: 10.1503/cmaj.1040752

19. Hadjihambi A, Arias N, Sheikh M, Jalan R (2018) Hepatic encephalopathy: a critical current review. Hepatol Int 12(Suppl 1):135-147. doi:10.1007/s12072-017-9812-3

20. Hussein AM, Bezu M, Korz V (2018) Evaluating Working Memory on a T-maze in Male Rats. Bioprotocol 8(14):e2930. doi:10.21769/BioProtoc.2930

21. Jaeger V, DeMorrow S, McMillin M (2019) The Direct Contribution of Astrocytes and Microglia to the Pathogenesis of Hepatic Encephalopathy. J Clin Transl Hepatol 28(4):352-361. doi:10.14218/JCTH.2019.00025. 7 ) .

22. Jayakumar AR, Rama Rao KV, Norenberg MD (2015) Neuroinflammation in hepatic encephalopathy: mechanistic aspects. J Clin Exp Hepatol (Suppl 1):S21-S28. doi:10.1016/j.jceh.2014.07.006

23. Khungar V, Poordad F (2012) Hepatic encephalopathy. Clin Liver Dis 16(2):301-320. doi:10.1016/j.cld.2012.03.009

24. Li S, Tan HY, Wang N, Zhang ZJ, Lao L, Wong CW, Feng Y (2015) The Role of Oxidative Stress and Antioxidants in Liver Diseases. Int J Mol Sci 16(11):26087-26124. doi:10.3390/ijms161125942

25. Liu Y, Tran DQ, Rhoads JM (2018) Probiotics in Disease Prevention and Treatment. J Clin Pharmacol 58(10):S164-S179. doi:10.1002/jcph.1121

26. Muramatsu K (1967) Direct Colorimetric Method for the Determination of Free Ammonia in Blood. Agr Biol Chem 31:3:301-308. doi:10.1080/00021369.1967.10858803 
27. Parekh PJ, Balart LA (2015 Aug) Ammonia and Its Role in the Pathogenesis of Hepatic Encephalopathy. Clin Liver Dis 19(3):529-537. doi:10.1016/j.cld.2015.05.002. Epub 2015 May 30. PMID: 26195206

28. Patel C, Patel P, Acharya S (2020) Therapeutic Prospective of a Spore-Forming Probiotic-Bacillus clausii UBBC07 Against Acetaminophen-Induced Uremia in Rats. Probiotics Antimicrob Proteins 12(1):253-258. doi:10.1007/s12602-019-09540-x

29. Prakash R, Mullen KD (2010) Mechanisms, diagnosis and management of hepatic encephalopathy. Nat Rev Gastroenterol Hepatol 7(9):515-525. doi:10.1038/nrgastro.2010.116

30. Ruiz-Larrea MB, Leal AM, Liza M, Lacort M, de Groot H (1994) Antioxidant effects of estradiol and 2hydroxyestradiol on iron-induced lipid peroxidation of rat liver microsomes. Steroids 59(6):383-388. doi:10.1016/0039-128x(94)90006-x

31. Savy N, Brossier D, Brunel-Guitton C, Ducharme-Crevier L, Du Pont-Thibodeau G, Jouvet P (2018) Acute pediatric hyperammonemia: current diagnosis and management strategies. Hepat Med 10:105-115. doi: 10.2147/HMER.S140711. Suárez I, Bodega G, Fernández B (2002) Glutamine synthetase in brain: effect of ammonia. Neurochem Int 41(2-3):123 - 42. doi: 10.1016/s01970186(02)00033-5

32. Swaminathan M, Ellul MA, Cross TJ (2018) Hepatic encephalopathy: current challenges and future prospects. Hepat Med 10:1-11. doi:10.2147/HMER.S118964

33. Vijitruth R, Liu M, Choi DY, Nguyen XV, Hunter RL, Bing G (2006) Cyclooxygenase-2 mediates microglial activation and secondary dopaminergic cell death in the mouse MPTP model of Parkinson's disease. J Neuroinflammation 3:6. doi:10.1186/1742-2094-3-6

34. You DD, Choi GS, Kim JM, Kwon CHD, Joh JW, Lee SK (2017) Long-term Outcomes for Liver Transplant Recipients in Terms of Hepatic Encephalopathy. Transplant Proc 49(6):1425-1429. doi:10.1016/j.transproceed.2017.02.054

\section{Tables}

Table 1 Effect of Probiotics, Lactobacillus plantarum UBLP40 and Bacillus clausiNBBBC07 combination in biochemical and neurochemical parameters against TAA-induced acute hepatic encephalopathy in rats 


\begin{tabular}{|lllll|}
\hline Parameters & Group I & Group II & Group III & Group IV \\
\hline AST & $59.83 \pm 6.61$ & $235 \pm 23.73^{\star \star \star \star a}$ & $172.83 \pm 8.06^{\star \star \star \star b}$ & $185.33 \pm 5.42^{\star \star \star \star b}$ \\
\hline ALT & $32 \pm 11.36$ & $70 \pm 6.41^{\star \star \star \star a}$ & $51.33 \pm 3.32^{\star \star \star \star b}$ & $52.83 \pm 1.94^{\star \star \star \star b}$ \\
\hline ALP & $88.66 \pm 32.81$ & $219.33 \pm 20.84^{\star \star \star \star a}$ & $178.16 \pm 11.58^{\star \star b}$ & $186.8 \pm 7.78^{\star \mathrm{b}}$ \\
\hline $\mathrm{NH}_{3}$ & $128.33 \pm 6.62$ & $245 \pm 5.32^{\star \star \star \star \mathrm{a}}$ & $170.33 \pm 5.35^{* \star \star \star b}$ & $137 \pm 9.7^{\star \star \star \star b}$ \\
\hline MDA & $125.3 \pm 3.38$ & $167.83 \pm 2.92^{\star \star \star \star a}$ & $153.16 \pm 3.48^{\star \star \star \star b}$ & $147 \pm 2.82^{\star \star \star \star b}$ \\
\hline GSH & $1.97 \pm 0.075$ & $0.833 \pm 0.039^{\star \star \star \star a}$ & $1.25 \pm 0.017^{\star \star \star \star b}$ & $1.55 \pm 0.023^{\star \star \star \star b}$ \\
\hline
\end{tabular}

The Values are expressed as mean \pm SD $(n=6)$, "a" TAA induced control significant compared to Normal control whereas "b" lactulose and probiotics treated group to TAA induced control group. ${ }^{\star \star \star *} \mathrm{p}<0.0001,{ }^{\star \star \star} \mathrm{p}<0.001,{ }^{\star \star} \mathrm{p}<0.01{ }^{\star} \mathrm{p} 0.05$.

\section{Figures}

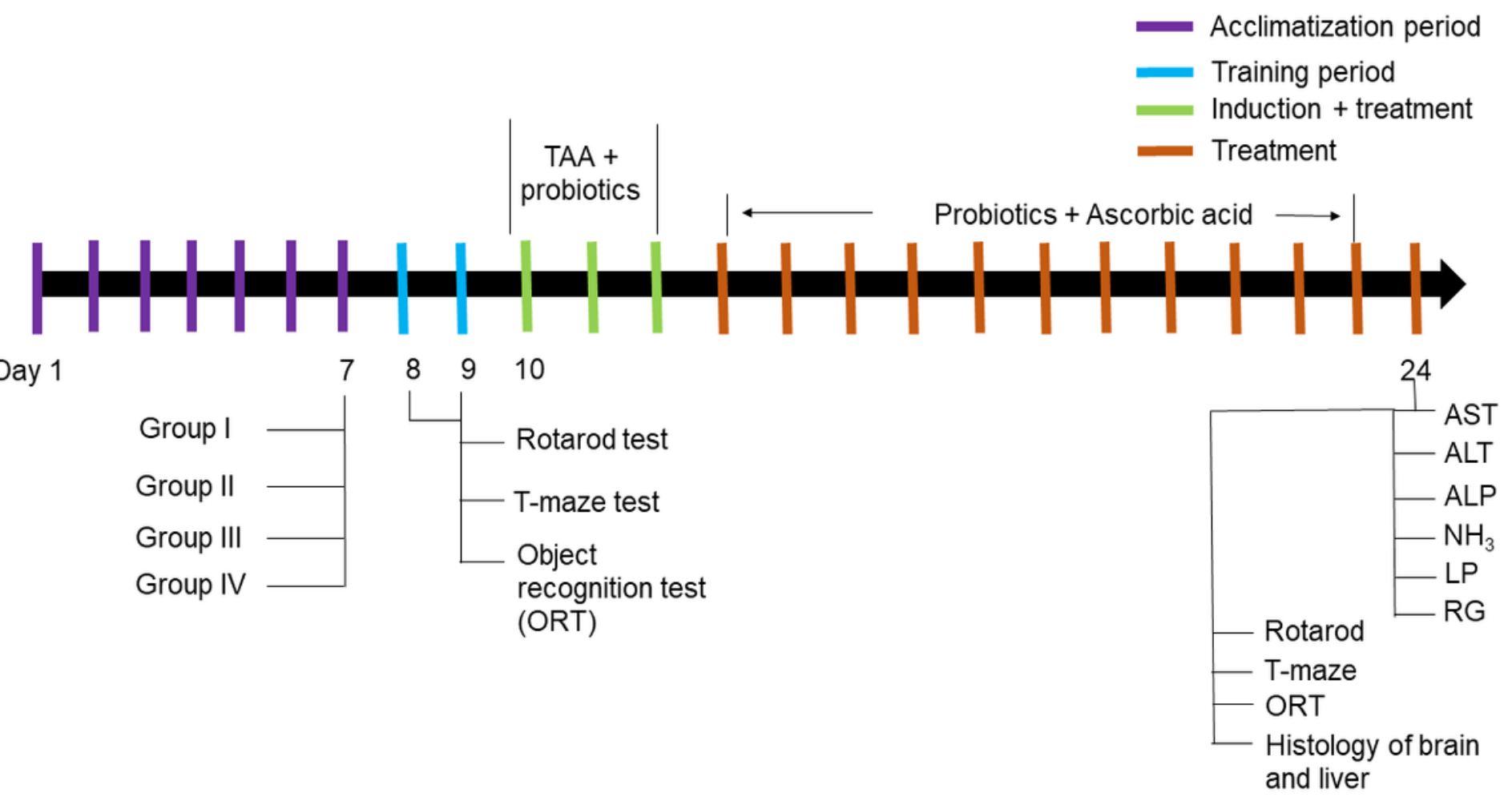

Figure 1

Experimental design. ALT-Alanine aminotransferase; AST-Aspartate aminotransferase; ALP-Alkaline Phosphatase (ALP); NH3- Ammonia; LP-Lipid Peroxidation; RG-Reduced glutathione 


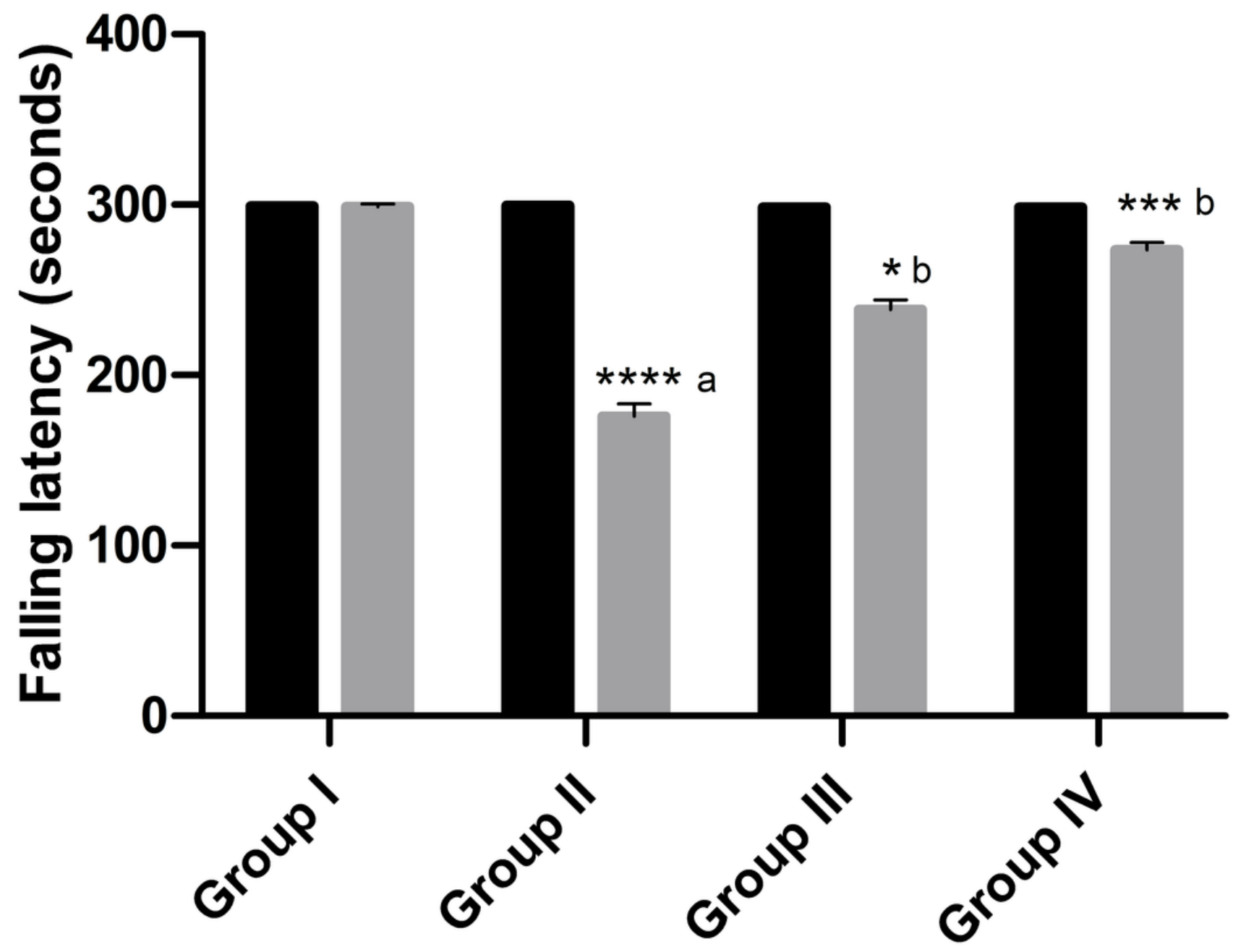

\section{Basal falling latency}

Final falling latency

Figure 2

Effect of Probiotics on motor coordination by Rotarod test in rats The values are calculated as mean \pm SD $(n=6)$, 'a' TAA induced control significant compared to Normal control whereas ' $b$ ' lactulose and probiotics treated group to TAA induced control group. ${ }^{\star \star \star \star} p<0.0001,{ }^{\star \star \star} p<0.001,{ }^{\star \star} p<0.01 * p 0.05$. 

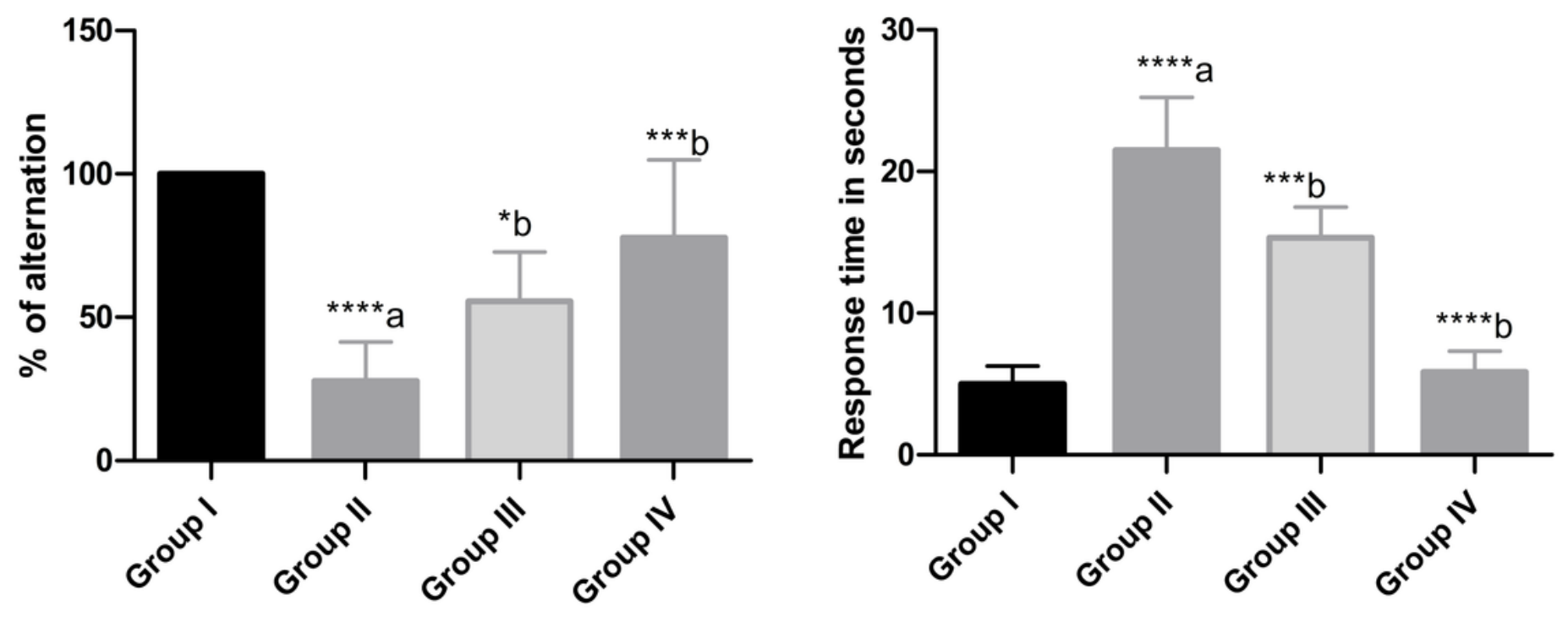

Figure 3

Effect of Probiotics on memory impairment by T-maze test in rats The values are calculated as mean \pm SD $(n=6)$, 'a' TAA induced control significant compared to Normal control whereas ' $b$ ' lactulose and probiotics treated group to TAA induced control group. ${ }^{\star \star \star \star} p<0.0001,{ }^{\star \star \star} p<0.001,{ }^{\star \star} \mathrm{p}<0.01{ }^{*} \mathrm{p} 0.05$. 


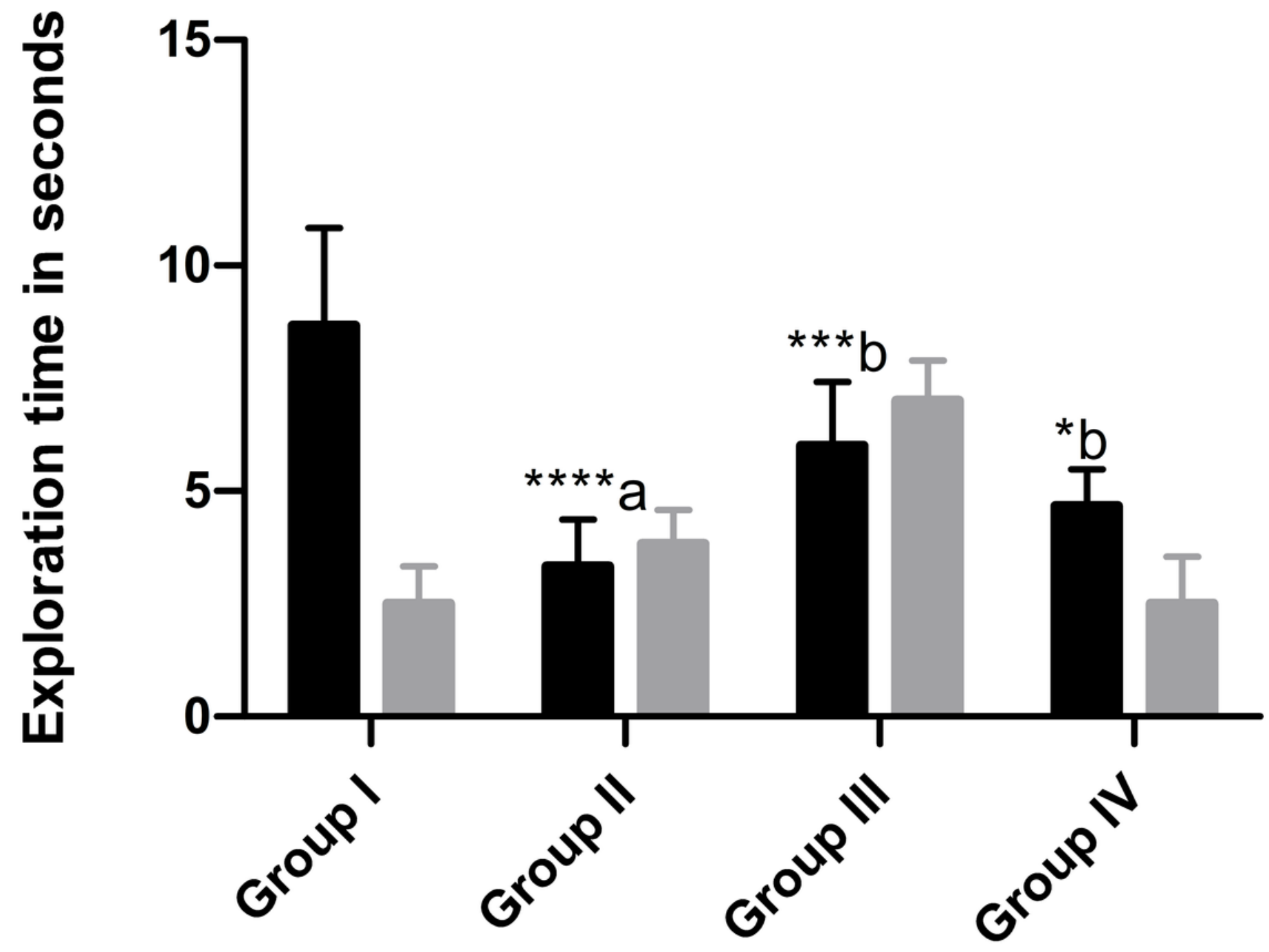

\section{New object}

\section{Familiar object}

Figure 4

Effect of Probiotic on memory impairment by Object recognition test in rats The values are calculated as mean \pm SD $(n=6)$, 'a' TAA induced control significant compared to Normal control whereas ' $b$ ' lactulose and probiotics treated group to TAA induced control group. ${ }^{\star \star \star *} p<0.0001,{ }^{\star \star *} p<0.001,{ }^{* \star} p<0.01$ *p0.05. 

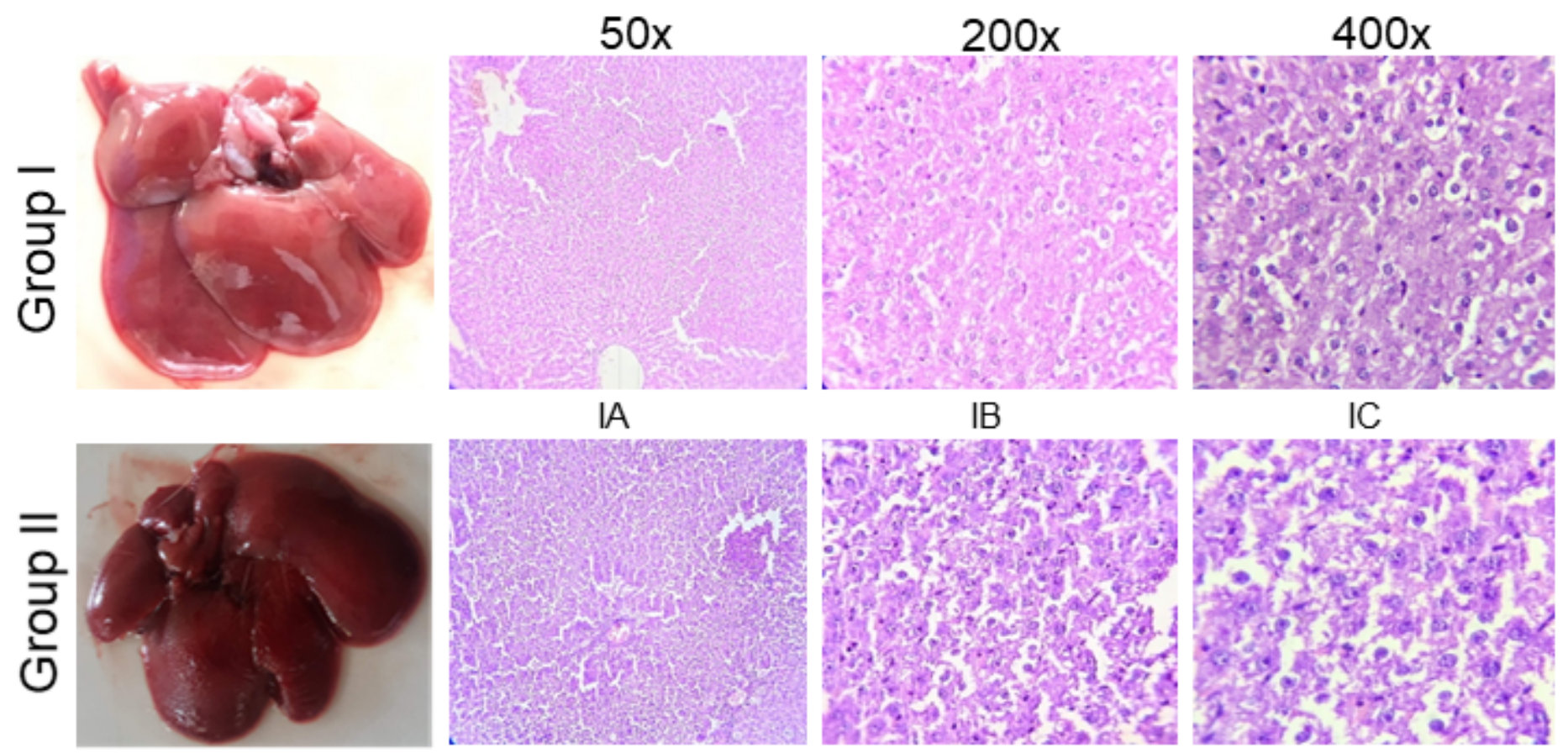

IIA
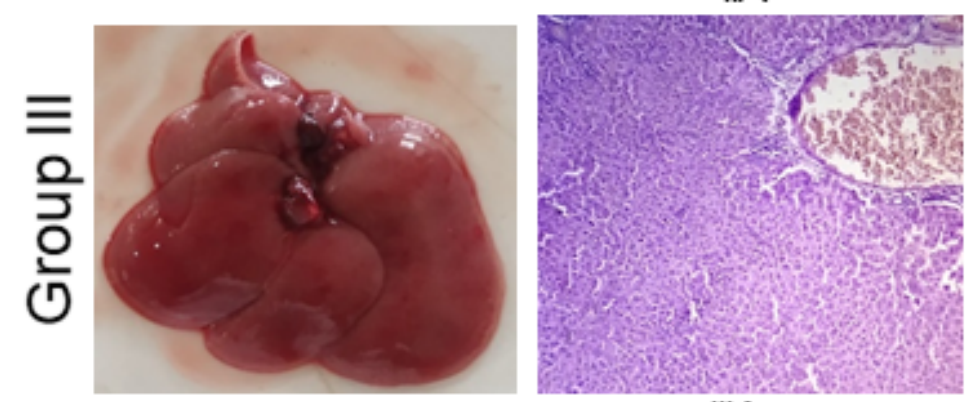

IIIA

IIB

IIC

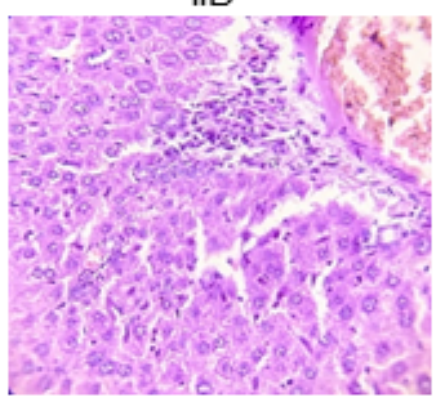

IIIB
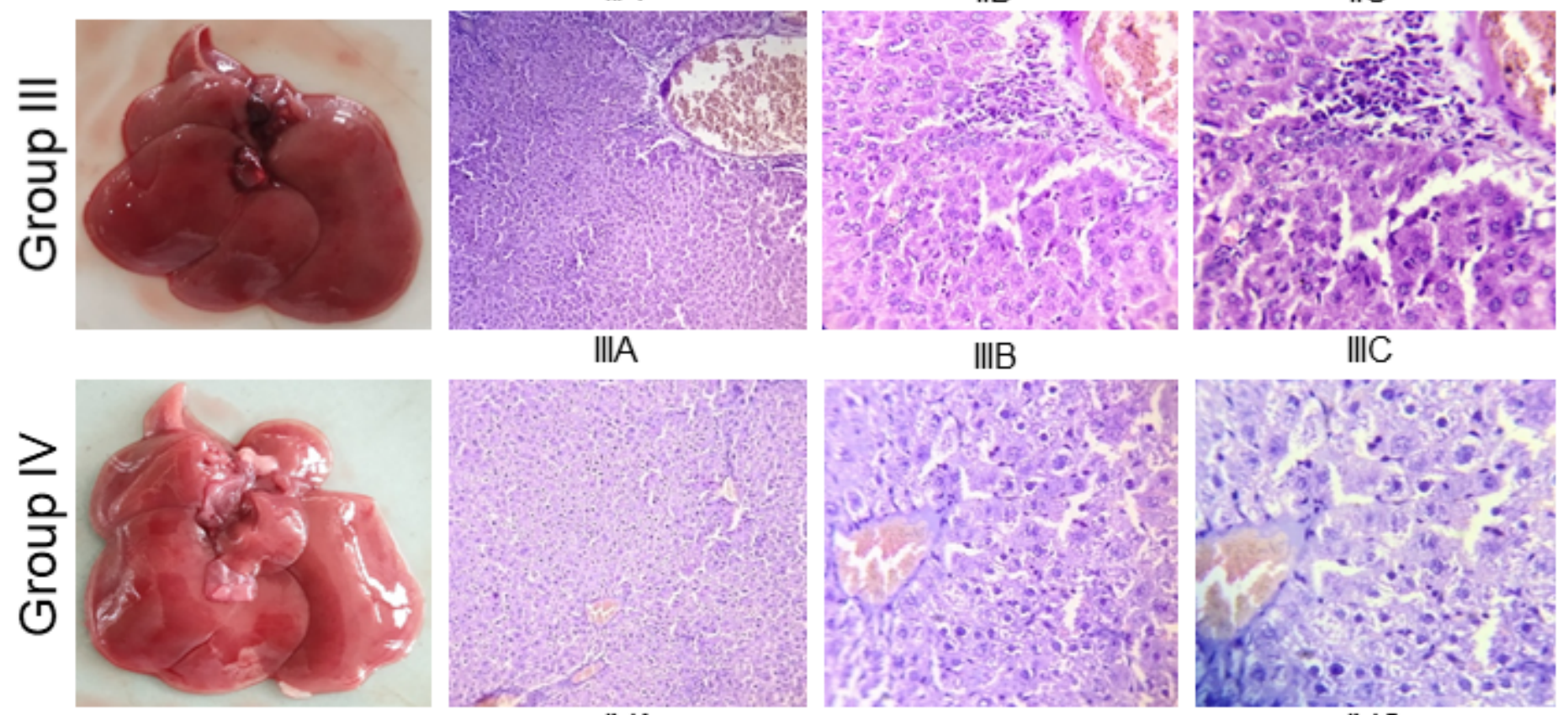

IVA

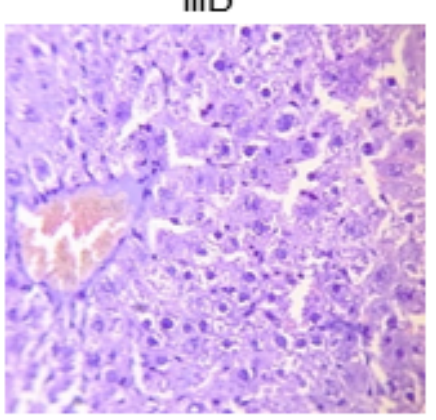

IVB
IIIC

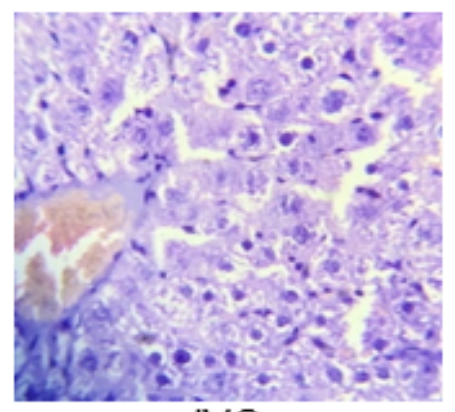

IVC

\section{Figure 5}

Histopathology study of effect of Probiotics on Liver against thioacetamide-induced acute hepatic encephalopathy in rats $(\times 50, \times 200, \times 400)$. 

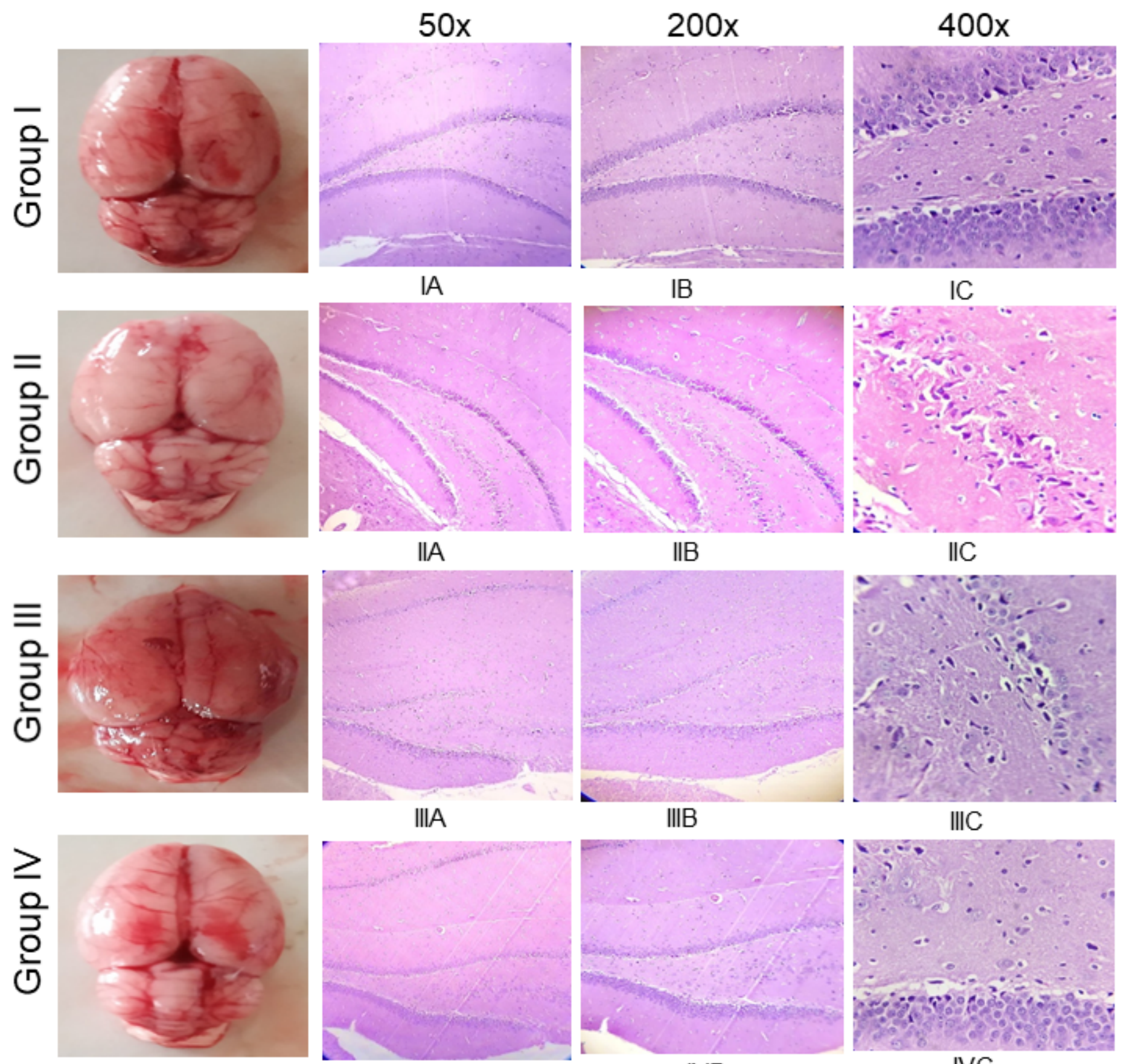

IVA

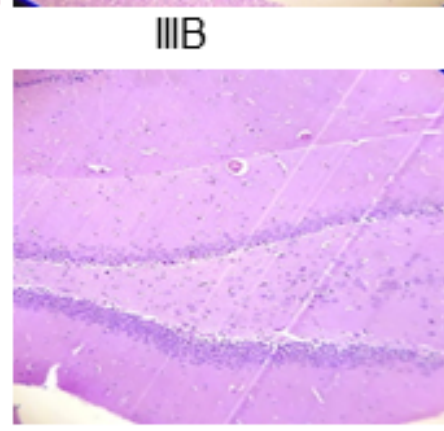

IVB

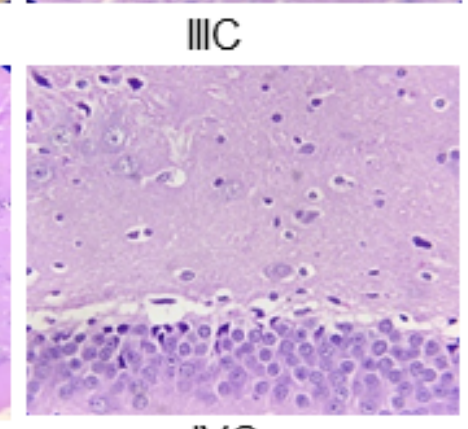

IVC

\section{Figure 6}

Histopathology study of effect of Probiotics on Brain hippocampus region against thioacetamideinduced acute hepatic encephalopathy in rats $(\times 50, \times 200, \times 400)$.

\section{Supplementary Files}

This is a list of supplementary files associated with this preprint. Click to download.

- SupplementaryFile1.docx 